\title{
Solarización de canteros en almácigos de cebolla para el control de malezas y enfermedades en Uruguay
}

\section{Soil solarization on onion beds for weed and disease control in Uruguay}
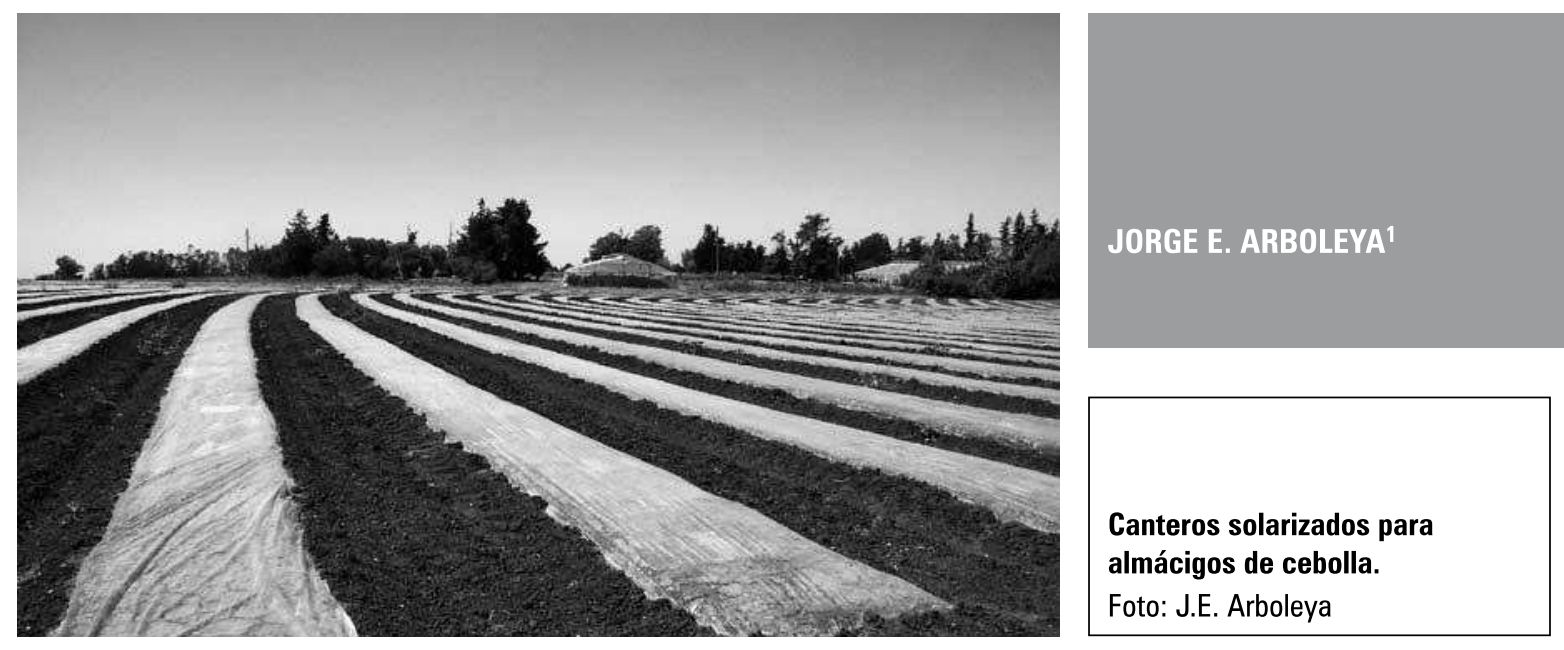

\section{RESUMEN}

Uruguay se ubica entre $35^{\circ}$ y $31^{\circ} \mathrm{S}$ y es un país con clima templado. La superficie ocupada por el sector granjero, principal proveedor de alimentos frescos, es de aproximadamente 1,8\% de la superficie agropecuaria del país. Hay 25.000 ha dedicadas a la horticultura sin incluir el cultivo de papa. El Instituto Nacional de Investigación Agropecuaria (INIA) tiene cinco estaciones experimentales en Uruguay. La estación INIA Las Brujas está localizada en el sur de Uruguay y sus acciones tienen por destinatario al productor granjero del sur. El Programa Nacional de Investigación Producción Hortícola abarca cinco áreas, dos de ellas "Control integrado de plagas a campo" y "Fisiología de cultivos". Durante las temporadas 2005-2006 se instalaron módulos demostrativos de solarización de canteros para el control de malezas en almácigos de cebolla. Se obtuvo una reducción significativa del número de malezas. Entre 2006 y 2008 se utilizaron dos espesores de polietileno transparente ultravioleta de 35 y $80 \mu \mathrm{m}$ y se los comparó con un testigo sin solarizar. Se obtuvo un excelente control de malezas sin existir diferencias estadísticas significativas entre ambos y sí con el testigo sin solarizar, al igual que en la calidad de las plántulas. En las parcelas solarizadas el nivel de amonio y nitratos en el suelo luego de la solarización fue mayor al testigo sin solarizar. La solarización también fue efectiva en reducir los daños de nematodos en plantines de cebolla en 2008.

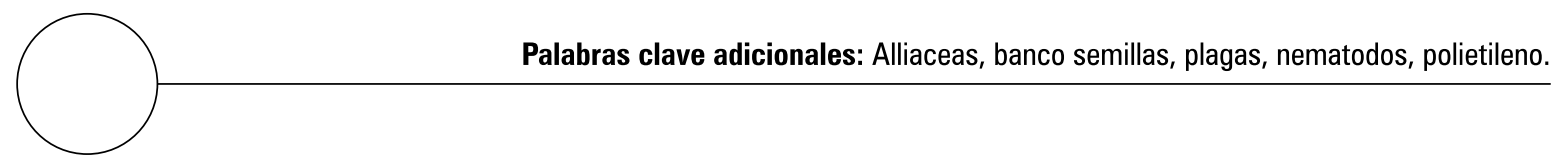

Estación Experimental Las Brujas, Instituto Nacional de Investigación Agropecuaria (INIA), Rincón del Colorado, Canelones (Uruguay). jarboleya@inia.org.uy 


\section{ABSTRACT}

Uruguay is located within $35^{\circ}$ and $31^{\circ}$ south latitude and is a country of temperate climate. The area occupied with agricultural sector, the main producer of fresh aliments, is about $1.8 \%$ of the agricultural area of the country. There are 25,000 ha of vegetable crops without including potato crop. The National Institute of Agricultural Research (INIA) has five experimental stations in the country. The INIA "Las Brujas" Research Station is located in the south of Uruguay. The National Research Program of Vegetable Crops includes five areas, two of which are integrated pest management in field and crop physiology. During the seasons 20052006, there were installed demonstrative modules of soil solarization to control the weeds in onion beds. The significant reduction in the number of weeds was obtained. During the years 2006 and 2008, there were utilized ultraviolet transparent polyethylene of two thickness grades of 35 and $80 \mu \mathrm{m}$ and compared with control without solarization. It was obtained an excellent control of weeds without the significant differences recorded among the thicknesses and with a significant difference recorded among the treatments and the control as well as for plantlet quality. In solarized treatments, the level of ammonium and nitrates in soil after solarization was the highest one in the control without solarization. The solarization was also effective in reducing the damages caused by nemathodes in plantlets of onion in 2008.

Additional key words: Alliaceae, seed bank, pests, nemathodes, polyethylene.

\section{Clima del Uruguay}

Uruguay se ubica entre $35^{\circ}$ y $31^{\circ} \mathrm{S}$ y es un país con clima templado. El relieve uruguayo está caracterizado por su escasa altitud. El punto más elevado del país es el cerro Catedral, con $514 \mathrm{msnm}$. La ausencia de sistemas orográficos importantes contribuye a que las variaciones espaciales de temperatura, precipitaciones y otros parámetros sean pequeñas (Severota, 1997).

La temperatura media anual es de $17,5^{\circ} \mathrm{C}$ variando desde unos $20^{\circ} \mathrm{C}$ en la zona noreste, hasta unos $16^{\circ} \mathrm{C}$ en la costa atlántica. Las isotermas tienen una orientación general del noreste al suroccidente y sus valores decrecen hacia el sudeste. Las temperaturas más altas se presentan en los meses de enero-febrero y las más bajas en junio-julio. Los cambios de temperaturas son fre- cuentes y pronunciados en cualquier época del año (El Clima del Uruguay, 2009).

Las lluvias totales medias anuales tienen su valor mínimo hacia el sur, sobre las costas del Río de la Plata, con casi $1.000 \mathrm{~mm}$ y su valor máximo hacia el noreste, en la frontera con Brasil, con $1.400 \mathrm{~mm}$.

Pese a esa distribución de valores medios, las precipitaciones en el Uruguay se caracterizan por su extremada irregularidad y variabilidad.

La humedad relativa media anual oscila entre 70 y $75 \%$, en todo el país. El mes más húmedo es julio (promedio $80 \%$ ) y el más seco es enero (promedio 65\%). La humedad relativa oscila alrededor de $45 \%$ al mediodía y superior a $90 \%$ en la madrugada. 
La superficie ocupada por el sector granjero es de $1,8 \%$ de la superficie agropecuaria del país. Abarca alrededor del $20 \%$ de los establecimientos rurales y de la población económicamente activa del medio rural. El valor bruto (VB) agropecuario de la producción de este sector representa el $15 \%$ del país. La horticultura contribuye con la tercera parte del mismo. Hay 25.000 ha dedicadas a la horticultura sin incluir el cultivo de papa. Este sector representa el principal proveedor de alimentos frescos para la población (Campelo y Arboleya, 2005).

Las dos zonas más importantes se ubican en el litoral norte y en la zona sur del país. Cerca de $90 \%$ de los productores son de carácter familiar. La orientación principal de la producción es hacia el mercado interno.

El Instituto Nacional de Investigación Agropecuaria (INIA) cuenta con cinco estaciones experimentales en Uruguay. La Estación Experimental INIA Las Brujas está localizada en el departamento de Canelones a $40 \mathrm{~km}$ de Montevideo. Las acciones de INIA Las Brujas tienen por destinatario principal al productor granjero de la zona sur.

\section{La producción de cebolla en Uruguay}

El cultivo de la cebolla es importante dentro de la granja nacional, ocupando el segundo lugar dentro de las hortalizas (tabla 1). Hay una superficie de cultivo cercana a las 2.000 ha anuales con un rendimiento promedio de 15 a $18 \mathrm{t} \mathrm{ha}^{-1}$. Esta producción es básicamente para consumo en fresco en el mercado interno (DIEA-MGAP, 2008).

En el departamento de Canelones, donde se ubica el INIA Las Brujas, se concentra la mayor área, donde se produce principalmente cebolla de estación con cosecha entre diciembre y febrero, aunque también se cultiva cebolla temprana. En el norte se ha desarrollado el cultivo aprovechando condiciones climáticas favorables para la producción temprana, logrando ingresar al mer- cado en los meses de octubre y noviembre. Gran parte del área cultivada se presenta en suelos de textura fina.

En la región sur, los suelos son de mayor fertilidad y la textura predominantemente es limosa a arcillo-limosa. Se presenta escaso drenaje interno, con un horizonte subsuperficial de permeabilidad baja. Por tanto, una limitante importante es el momento oportuno para el laboreo del suelo, que suele ser de pocos días al mes y exige una permanente atención a las condiciones climáticas.

En la zona norte, fundamentalmente en el departamento de Salto, la siembra se realiza en marzo, el trasplante en mayo-junio y la cosecha de finales de septiembre a noviembre, según el cultivar.

En la región sur, los cultivos se destinan principalmente a la conservación y abastecimiento del mercado durante un periodo que comprende de febrero a agosto. También se cultiva cebolla temprana y para venta entre noviembre y marzo. El área cultivada por productor es de 1,5 a 2,0 ha, existiendo una amplia variabilidad con áreas menores hasta cultivos de 15 a 25 ha.

Las exportaciones no superan el $5 \%$ de la producción nacional y en general los volúmenes importados superan ampliamente a las exportaciones.

En el presente artículo, aparte de la información sobre el clima del Uruguay, de algunas caracte-

\section{Tabla 1. Principales rubros hortícolas, número de productores y superficie por cultivo.}

\begin{tabular}{|l|r|r|}
\multicolumn{1}{|c|}{ Cultivos } & $\begin{array}{c}\text { Productores } \\
\text { (No.) }\end{array}$ & $\begin{array}{c}\text { Superficie } \\
\text { (ha) }\end{array}$ \\
\hline Boniato (batata) & 1.431 & 1.761 \\
\hline Cebolla & 1.290 & 1.709 \\
\hline Zanahoria & 521 & 1.597 \\
\hline Zapallo Kabutiá & 634 & 2.175 \\
\hline Tomate industria & 667 & 483 \\
\hline Tomate mesa & 410 & 234 \\
\hline
\end{tabular}

Fuente: DIEA-MGAP, 2008. 
rísticas importantes del sector granjero y de la producción de cebolla, se detallan los principales resultados de la investigación realizada en los almácigos de cebolla con la técnica de la solarización.

\section{La investigación en horticultura y de cebolla en Uruguay}

El Programa Nacional de Investigación Producción Hortícola abarca cinco áreas y entre ellas "Control integrado de plagas a campo" y "Fisiología de cultivos". Dentro de este último se encuentra una línea de investigación "Ajuste de alternativas en el control de malezas en cultivos hortícolas, como almácigos de cebolla". Desde el 2007 existe una línea de investigación aplicada con financiamiento del Banco Mundial titulado "Métodos amigables con el medio ambiente para el manejo de malezas, enfermedades, plagas y residuos de los establecimientos". En esta línea también participan la Facultad de Agronomía (Fagro) y la Dirección General de la Granja (Digegra). En estos dos proyectos se ha trabajado en cebolla en las temáticas de control de malezas y de plagas utilizando la técnica de la solarización.

La solarización en su forma actual fue descrita por Katan et al. (1976) y Davis (1991) menciona que la solarización es efectiva en el control de hongos, bacterias, malezas y nematodos. Importante para el caso de la germinación de semillas de las malezas es que más arriba de un nivel óptimo de temperatura ocurre una declinación a medida que el calor se aproxima al límite letal y la semilla se daña (Hartmann et al., 2001). Para el control del moho blanco (Sclerotinia spp.) en lechuga, Gil et al. (2009) encontaron efectos favorables de la solarización con una cobertura de plástico, manteniendo temperaturas de $45,3^{\circ} \mathrm{C}$ en promedio.

En la década de 1980 se experimentó con la solarización en el norte del Uruguay, donde la temperatura del aire es en promedio $5^{\circ} \mathrm{C}$ superior a la de la zona sur y en algunos lugares los suelos presentan una textura más liviana que los del sur, por lo que se logran incrementos térmicos de significancia al realizar la solarización. Desde 2004 se realizaron experiencias de solarización de canteros para almácigos de cebolla en la zona sur del país en el Centro Regional Sur (CRS) de Fagro, en suelos de textura pesada. Los resultados obtenidos mostraron que se obtuvo una reducción del número de malezas de 850 plantas $/ \mathrm{m}^{2}$ en el cantero no solarizado a 12 plantas $/ \mathrm{m}^{2}$ en los almácigos de cebolla con la solarización. Este efecto se mantuvo $100 \mathrm{~d}$ después de levantar el polietileno de los almácigos.

\section{Módulos demostrativos}

Desde diciembre de 2005, INIA, Digegra y Fagro realizaron módulos demostrativos en tres zonas del sur del Uruguay (Campelo et al., 2006). En las solarizaciones se utilizó polietileno ultravioleta transparente de un espesor de $80 \mu \mathrm{m}$ (PE UV transp. $80 \mu \mathrm{m}$ ). Se compararon un tratamiento sin solarizar y el solarizado con y sin riego luego de colocar el PE. Se instalaron registradores automáticos de temperatura, tipo Kooltrak, programados para toma de datos cada $2 \mathrm{~h}$ a 5 y $20 \mathrm{~cm}$ de profundidad. Se evaluó el número de malezas por superficie de almácigos contabilizando las mismas en un cuadrante de $0,40 \times 0,40 \mathrm{~m}$ (Arboleya et al., 2008).

En las figuras 1, 2A y $2 \mathrm{~B}$ se muestran las variaciones de la temperatura obtenida en un suelo de textura pesada (arcillosa) a 5 y $20 \mathrm{~cm}$ de profundidad, respectivamente, en el suelo solarizado y en el no solarizado en Las Violetas, departamento de Canelones.

Si bien a $20 \mathrm{~cm}$ de profundidad las temperaturas en los canteros solarizados fueron superiores a las de los no solarizados, fueron menores que a 5 $\mathrm{cm}$ de profundidad. Por ello la mayor acción de la temperatura ocurre en los primeros centímetros de suelo y de allí la importancia de no llevar a superficie las capas de abajo del suelo para impedir que semillas que no hubieran sido afectadas por el efecto de la solarización pudieran germinar. 

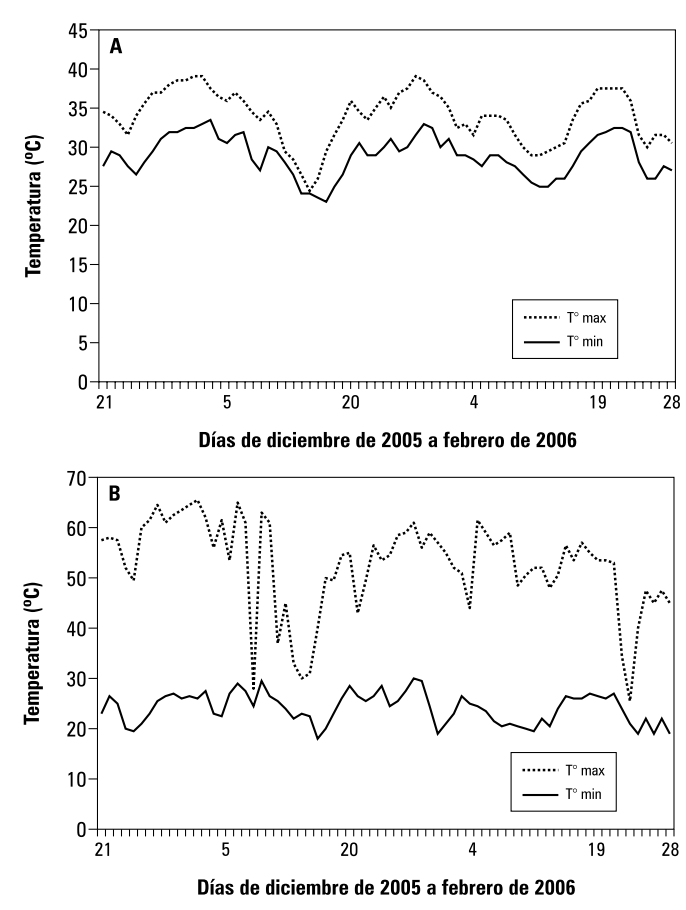

Figura 1. Temperaturas máximas y mínimas a $5 \mathrm{~cm}$ de profundidad, suelo no solarizado (A) y suelo solarizado (B). Las Violetas, Canelones.

En las figuras $3 \mathrm{~A}$ y $3 \mathrm{~B}$ se aprecian las temperaturas máximas y mínimas a 5 y $20 \mathrm{~cm}$ de profundidad, respectivamente, en el suelo no solarizado y en el solarizado con textura media a liviana del departamento de Montevideo. La figura 4 muestra los datos de temperatura máxima y mínima a $20 \mathrm{~cm}$ de profundidad para el suelo solarizado.

De los resultados obtenidos en Rincón del Cerro, a $5 \mathrm{~cm}$ de profundidad, se puede afirmar que también existieron diferencias importantes entre el cantero solarizado y el no solarizado, llegando hasta más de $50^{\circ} \mathrm{C}$ la temperatura en el solariza-

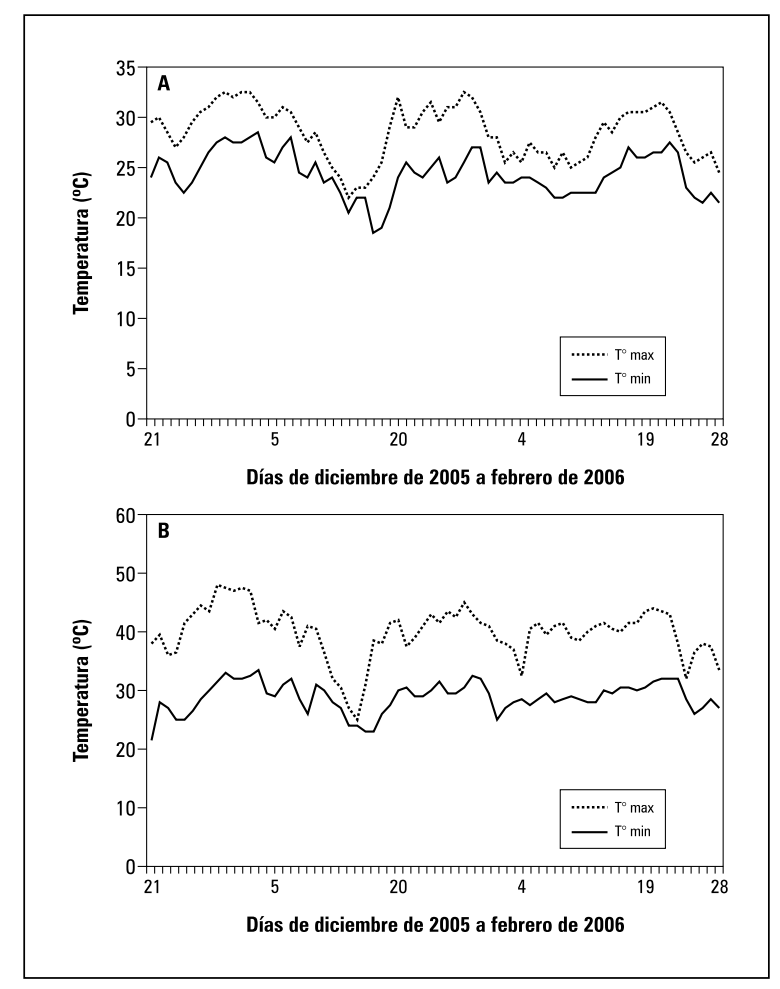

Figura 2. Temperaturas máximas y mínimas a $20 \mathrm{~cm}$ de profundidad, suelo no solarizado $(A)$ y suelo solarizado (B). Las Violetas, Canelones.

do. Las temperaturas a $20 \mathrm{~cm}$ de profundidad siguieron la misma tendencia que para la localidad de Las Violetas, es decir menores que a $5 \mathrm{~cm}$.

Los resultados de la evaluación del control de malezas en las tres localidades se resumen en la tabla 2.

Las conclusiones de estos módulos fueron:

- La técnica de la solarización fue efectiva en el control de malezas en canteros para almácigos de cebolla tanto en Brisas del Plata, en Las

Tabla 2. Número de malezas/m² de almácigos en Brisas del Plata, Colonia; Las Violetas, Canelones, y Rincón del Cerro, Montevideo.

\begin{tabular}{|l|c|c|c|}
\hline \multicolumn{1}{|c|}{ Tratamiento } & Brisas del Plata & Las Violetas & Rincón del Cerro \\
\hline Suelo no solarizado & 760 & 350 & 6.934 \\
\hline Suelo solarizado y regado & 3 & 6 & -- \\
\hline Suelo solarizado, sin riego posterior & 0 & 25 & 118 \\
\hline
\end{tabular}

${ }^{1}$ El cantero no solarizado recibió tres aplicaciones de glifosato entre enero y abril de 2006. 
Violetas y en Rincón del Cerro. Estos resultados confirman lo encontrado por Mallek et al. (2007) quienes reportan un control efectivo en la germinación de semillas de varias especies de malezas a $39^{\circ} \mathrm{C}$, comparado con $23^{\circ} \mathrm{C}$.

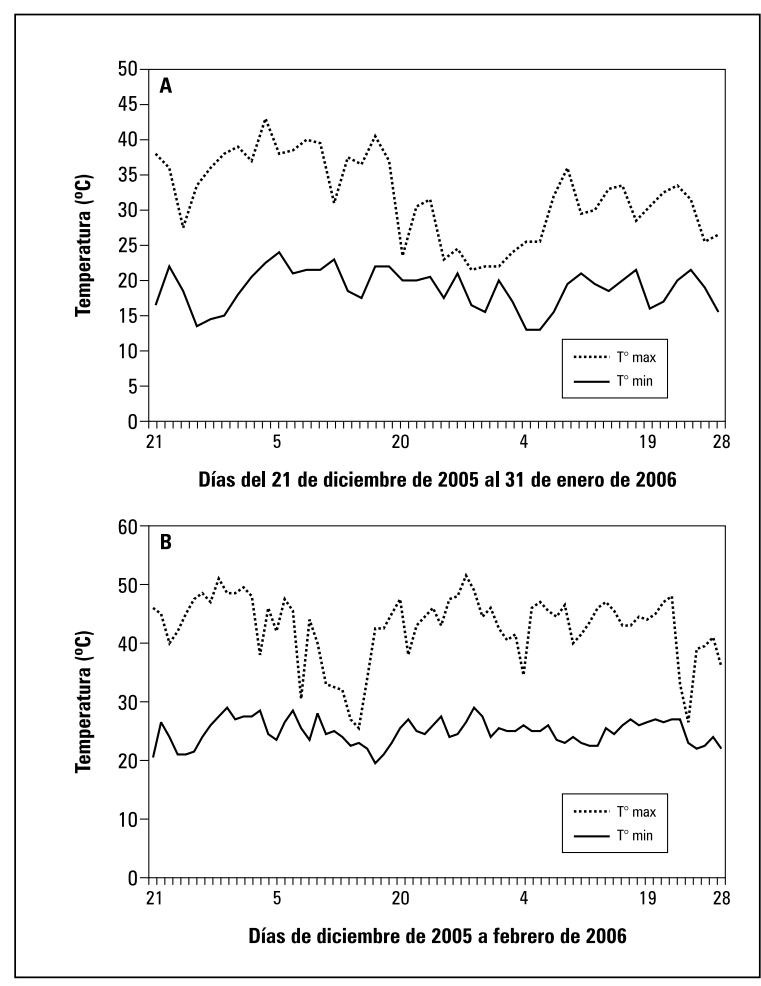

Figura 3. Temperaturas máximas y mínimas a 5 y $20 \mathrm{~cm}$ de profundidad, suelo no solarizado $(A)$ y suelo solarizado (B). Rincón del Cerro, Montevideo.

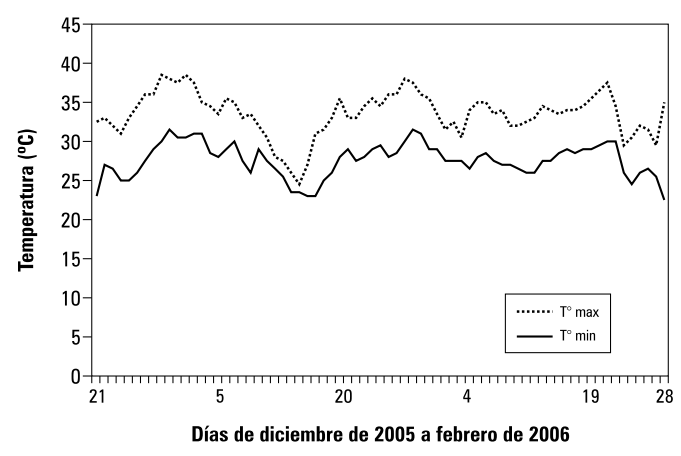

Figura 4. Temperaturas máximas y mínimas a $20 \mathrm{~cm}$ de profundidad, suelo solarizado. Rincón del Cerro, Montevideo.
- A pesar de que las temperaturas del mes de enero de 2006 fueron en algún momento más bajas a las normales, los registros de las máximas de fines del mes de diciembre y las registradas en enero y febrero fueron suficientes para lograr el objetivo de disminuir significativamente la infestación de malezas en los canteros solarizados. Esto confirma los resultados obtenidos en el CRS-Fagro en 2004-2005.

- El efecto del control estuvo relacionado al grado de infestación de semillas de malezas del suelo en cada caso, pero se dieron grandes diferencias con el cantero no solarizado en todos los casos.

- La solarización realizada en los canteros que no tenían cinta de riego, pero que habían sido regados hasta capacidad de campo antes de colocarse el PE para la solarización, fue efectiva en el control de malezas en relación al cantero no solarizado.

\section{Efecto de la solarización con el uso de estiércol, compost y microorganismos efectivos sobre el banco de malezas y calidad de la plántula}

En 2006-2007 se instaló un experimento en la localidad de Santa Rosa, cercana a la zona productora de cebolla de Canelones (Campelo et al., 2007). En esta zona es común el uso de estiércol de ave en cuyo piso se coloca cáscara de arroz. Se utilizó este material y un compost de la Intendencia Municipal de Montevideo (IMM). Además, se aplicaron los microorganismos efectivos (EM).

La cobertura del suelo se realizó el 13 de diciembre de 2006 con PE UV transp. $35 \mu \mathrm{m}$ y se mantuvo hasta el momento de la siembra. Se plantó el cultivar Pantanoso del Sauce-CRS en canteros de cuatro filas el 27 de abril de 2007 con 4 g de semilla $/ \mathrm{m}^{2}$. El diseño experimental fue de bloques al azar con tres repeticiones. Se evaluó el número de malezas por superficie de cantero, a los 40 y 60 d después de la siembra (dds), utili- 
zando la misma metodología anterior. Se tomaron muestras de suelo a $15 \mathrm{~cm}$ de profundidad a los $65 \mathrm{~d}$ después de retirar el PE, para determinar el contenido de amonio y de nitratos. A los 94 dds se tomó una muestra de 10 plántulas representativas de cada parcela y se evaluó el largo desde el disco basal a la hoja más larga, el diámetro del falso tallo, el peso fresco, peso seco y se determinó el contenido de $\mathrm{N}$ foliar.

Se colocaron registradores para la toma de la temperatura del suelo, en este caso a una profundidad de $10 \mathrm{~cm}$. En la figura 5 se grafican las temperaturas máximas y mínimas a $10 \mathrm{~cm}$ de profundidad en el suelo solarizado (A) y en el no solarizado (B).

El 7 (40 dds) y el 27 de julio (60 dds) se determinó, aparte del peso fresco y seco, el número de malezas y los resultados se expresan en número de malezas $/ \mathrm{m}^{2}$ de almácigo (tabla 3).

Los tratamientos que recibieron incorporación de estiércol (T4) y compost de IMM (T6) sin solarizar presentaron mayor cantidad de malezas que el tratamiento testigo (T1), aunque estadísticamente no fueron significativamente diferentes. Debe tenerse en cuenta que el coeficiente de variación fue muy alto (46\%) y debido a ello posiblemente no se detectaron esas diferencias. Sin embargo en promedio habían 68 malezas en el T1 en comparación con 106 en el T4 y 110 para el T6, en las dos fechas evaluadas. Esto reafirma aún más la importancia de solarizar el material orgánico a agregar ya que puede ser una potencial fuente de incorporación de malezas.

Las malezas predominantes en las parcelas del experimento fueron: capiquí (Stellaria media), mastuerzo (Coronopus didymus), perejilillo (Fumaria spp.), pega lana (Picris echoides), bowlesia (Bowlesia incana), falsa ortiga (Stachis arvensis), manzanilla (Matricaria chamomilla), sanguinaria (Poligonum aviculare), rábano (Raphanus sp.), cerraja (Sonchus oleraceus) y trébol de campo (Trébol spp.). Esta última predominó en los tratamientos solarizados.

La tendencia observada, en cuanto a número de malezas, también fue similar a la del peso de malezas por parcela (tabla 3). Los tratamientos no solarizados presentaron significativamente mayor peso fresco y seco de malezas en relación a los solarizados.

El análisis del contenido de nitrato y amonio del suelo de las parcelas indicó que los tratamientos solarizados tuvieron significativamente mayor contenido de nitratos que las no solarizadas, con excepción del T4 (tabla 4).

Tabla 3. Número de malezas del almácigo a los 40 y 60 dds y el peso fresco y seco a los 94 dds de la cebolla.

\begin{tabular}{|c|c|c|c|c|}
\hline \multirow{2}{*}{ Tratamientos } & \multicolumn{2}{|c|}{ No. de malezas } & \multirow{2}{*}{$\begin{array}{c}\text { Peso fresco } \\
\text { (g) }\end{array}$} & \multirow{2}{*}{$\begin{array}{c}\text { Peso seco } \\
\text { (g) }\end{array}$} \\
\hline & $40 \mathrm{dds}$ & $60 \mathrm{dds}$ & & \\
\hline 1 No solarizado & 65 a & $71 \mathrm{~b}$ & $21,00 \mathrm{~b}$ & $4,00 \mathrm{bc}$ \\
\hline 2 Solarizado (PE UV transp. $35 \mu \mathrm{m}$ ) & $0 \mathrm{~b}$ & $2 \mathrm{c}$ & $0,90 \mathrm{c}$ & $0,14 \mathrm{c}$ \\
\hline 3 Solarizado +10 t estiércol antes de solarizar & $7 \mathrm{~b}$ & $8 \mathrm{c}$ & $0,14 \mathrm{c}$ & $0,07 \mathrm{c}$ \\
\hline 4 No solarizado +10 t estiércol & 104 a & $108 \mathrm{ab}$ & $47,00 \mathrm{a}$ & 8,70 a \\
\hline 5 Solarizado +5 t compost IMM antes de solarizar & $7 \mathrm{~b}$ & $13 \mathrm{c}$ & $2,74 \mathrm{c}$ & $0,50 \mathrm{c}$ \\
\hline 6 No solarizado +5 t compost IMM a la siembra & 106 a & 114 a & $33,00 \mathrm{ab}$ & $7,40 a b$ \\
\hline 7 Solarizado $+200 \mathrm{~L} \mathrm{ha}^{-1} \mathrm{EM}$ a la siembra & $13 \mathrm{~b}$ & $17 \mathrm{c}$ & $0,87 \mathrm{c}$ & $0,14 c$ \\
\hline 8 Solarizado +10 t estiércol $+30 \mathrm{~L} \mathrm{ha}^{-1} \mathrm{EM}$ antes de colocar el PE & $0 \mathrm{~b}$ & $38 \mathrm{c}$ & $0,01 \mathrm{c}$ & $0,01 \mathrm{c}$ \\
\hline CV (\%) & 49 & 28 & 78 & 89 \\
\hline LSD & 32 & 16 & 18,00 & 5,70 \\
\hline
\end{tabular}

Los promedios con letras distintas indican diferencia significativa según la prueba de $\operatorname{LSD}(P \leq 0,01)$. 

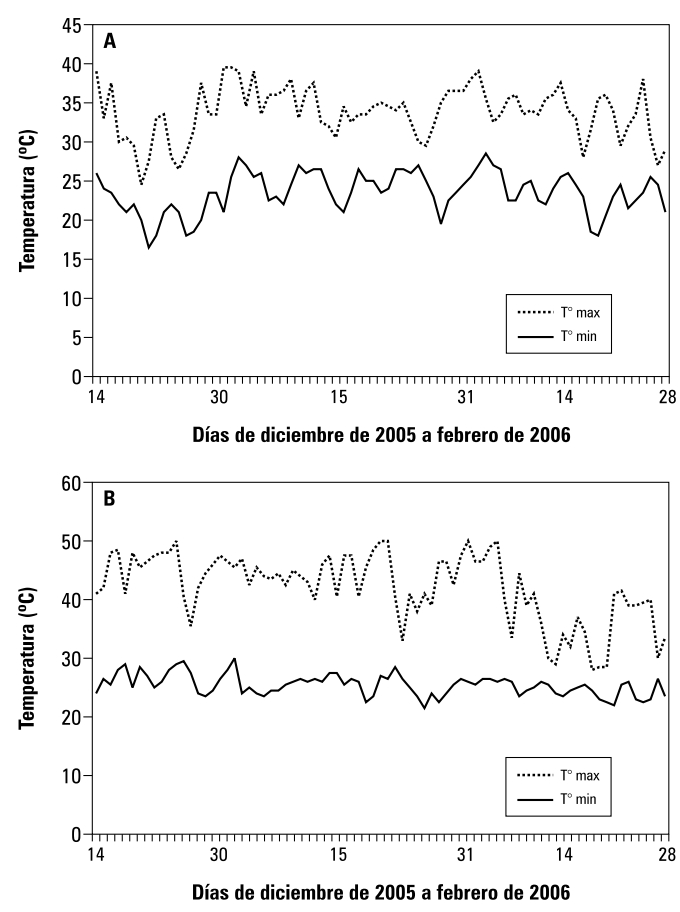

Figura 5. Temperaturas máximas y mínimas a $10 \mathrm{~cm}$ de profundidad, suelo no solarizado (A) y solarizado (B), Santa Rosa, Canelones 20062007.
Los tratamientos solarizados presentaron una mayor altura de plántula que los no solarizados (tabla 4). El peso fresco y seco de las plántulas de los tratamientos no solarizados fue significativamente menor que en el de los plantines solarizados (tabla 5).

Las plántulas del tratamiento solarizado (T2), del solarizado con previo agregado de estiércol (T3) y el tratamiento con agregado de estiércol, de EM y solarizado (T8) fueron las que presentaron los mayores valores $(3,8 \%)$ de $\mathrm{N}$ foliar (tabla 5). El tratamiento con agregado de EM a la siembra y que había sido solarizado (T7) no difirió estadísticamente de los anteriores con 3,4\% de N. Podría ser que los EM estuvieran favoreciendo una mayor actividad microbiana y de mineralización.

Los tratamientos testigo, el no solarizado con agregado de estiércol de parrillero, el no solarizado y agregado de compost y el solarizado con agregado de compost tuvieron un contenido significativamente menor de $\mathrm{N}$ foliar $(2,90 ; 2,82$; 3,03 y 3,06 , respectivamente) en comparación con los anteriores.

Tabla 4. Nitratos y amonio en suelo 65 dias despues de levantar el polietileno de la solarizacion y altura de la plántula y diámetro del falso tallo a 101 dds de la cebolla.

\begin{tabular}{|l|c|c|c|c|}
\hline \multicolumn{1}{|c|}{ Tratamientos } & $\begin{array}{c}\text { Nitratos } \\
\left(\mathrm{mg} \mathrm{kg}^{-1}\right)\end{array}$ & $\begin{array}{c}\text { Amonio } \\
\left(\mathrm{mg} \mathrm{kg}^{-1}\right)\end{array}$ & $\begin{array}{c}\text { Altura } \\
\text { plántula } \\
(\mathrm{cm})\end{array}$ & $\begin{array}{c}\text { Diámetro } \\
\text { falso tallo } \\
(\mathrm{mm})\end{array}$ \\
\hline 1 No solarizado & $4,5 \mathrm{~b}$ & $5,7 \mathrm{~d}$ & $19 \mathrm{c}$ & $3,8 \mathrm{c}$ \\
\hline 2 Solarizado (PE UV transp. 35 $\mu \mathrm{m})$ & $12,5 \mathrm{ab}$ & $22,2 \mathrm{bc}$ & $24 \mathrm{ab}$ & $5,1 \mathrm{a}$ \\
\hline 3 Solarizado + 10 t estiércol antes de solarizar & $19,6 \mathrm{a}$ & $33,9 \mathrm{ab}$ & $24 \mathrm{ab}$ & $5,1 \mathrm{a}$ \\
\hline 4 No solarizado + 10 t estiércol & $12,6 \mathrm{ab}$ & $8,0 \mathrm{~cd}$ & $22 \mathrm{bc}$ & $5,0 \mathrm{ab}$ \\
\hline 5 Solarizado + 5 t compost IMM antes de solarizar & $16,2 \mathrm{ab}$ & $24,4 \mathrm{ab}$ & $23 \mathrm{ab}$ & $5,0 \mathrm{a}$ \\
\hline 6 No solarizado + 5 t compost IMM a la siembra & $4,8 \mathrm{~b}$ & $6,3 \mathrm{~d}$ & $19 \mathrm{c}$ & $4,1 \mathrm{bc}$ \\
\hline 7 Solarizado + 200 L ha ${ }^{-1}$ EM a la siembra & $23,2 \mathrm{a}$ & $25,3 \mathrm{ab}$ & $24 \mathrm{ab}$ & $4,9 \mathrm{ab}$ \\
\hline 8 Solarizado + 10 t estiércol + 30 L ha-1 EM antes de colocar el PE & $17,3 \mathrm{ab}$ & $38,9 \mathrm{a}$ & $26 \mathrm{a}$ & $5,0 \mathrm{a}$ \\
\hline CV (\%) & 43 & 29 & 9,7 & 29 \\
\hline LSD & 10,4 & 10,6 & 3,5 & 10,6 \\
\hline
\end{tabular}

Los promedios con letras distintas indican diferencia significativa según la prueba de $\operatorname{LSD}(P \leq 0,01)$. 
Tabla 5. Contenido de nitrógeno foliar a 94 dds y peso fresco y seco de plántulas a 101 dds de la cebolla.

\begin{tabular}{|l|c|c|c|}
\hline \multicolumn{1}{|c|}{ Tratamientos } & $\begin{array}{c}\text { Nitrógeno } \\
\text { foliar }(\%)\end{array}$ & $\begin{array}{c}\text { Peso fresco } \\
\text { (g/10 plántulas) }\end{array}$ & $\begin{array}{c}\text { Peso seco } \\
\text { (g/10 plántulas) }\end{array}$ \\
\hline 1 No solarizado & $2,90 \mathrm{~b}$ & $9 \mathrm{~b}$ & $1,3 \mathrm{c}$ \\
\hline 2 Solarizado (PE UV transp. 35 $\mu \mathrm{m})$ & $3,81 \mathrm{a}$ & $19 \mathrm{a}$ & $2,4 \mathrm{ab}$ \\
\hline 3 Solarizado + 10 t estiércol antes de solarizar & $3,83 \mathrm{a}$ & $19 \mathrm{a}$ & $2,3 \mathrm{ab}$ \\
\hline 4 No solarizado + 10 t estiércol & $2,82 \mathrm{~b}$ & $14 \mathrm{ab}$ & $1,8 \mathrm{bc}$ \\
\hline 5 Solarizado + 5 t compost IMM antes de solarizar & $3,06 \mathrm{~b}$ & $19 \mathrm{a}$ & $2,5 \mathrm{a}$ \\
\hline 6 No solarizado + 5 t compost IMM a la siembra & $3,03 \mathrm{~b}$ & $9 \mathrm{~b}$ & $1,2 \mathrm{c}$ \\
\hline 7 Solarizado + 200 L ha-1 EM a la siembra & $3,41 \mathrm{ab}$ & $17 \mathrm{a}$ & $2,1 \mathrm{ab}$ \\
\hline 8 Solarizado + 10 t estiércol + 30 L ha-1 EM antes de colocar el PE & $3,77 \mathrm{a}$ & $18 \mathrm{a}$ & $2,2 \mathrm{ab}$ \\
\hline CV (\%) & 14 & 21 & 19 \\
\hline LSD & 0,67 & 3,5 & 0,66 \\
\hline
\end{tabular}

Los promedios con letras distintas indican diferencia significativa según la prueba de $\operatorname{LSD}(P \leq 0,01)$.

\section{Efecto de dos espesores de polietileno sobre el control de malezas y calidad de la plántula}

En 2006-2007 y en 2007-2008 en el campo experimental de INIA Las Brujas se comparó el efecto de dos espesores de PE UV transp. 35 y $80 \mu \mathrm{m}$ sobre el control de malezas y sobre el desarrollo de los plantines (Arboleya et al., 2008). La colocación del plástico se realizó el 17 de diciembre de 2006 y se mantuvo hasta el momento de la siembra de los almácigos. Se plantó el cultivar Pantanoso del Sauce CRS en canteros de cuatro filas el 30 de abril de 2007. Se realizaron evaluaciones similares a las descritas en el punto anterior.

El 4 de junio (36 dds) se determinó el número de malezas en un cuadrante de 0,40 × 0,40 m y se calculó el número de malezas $/ \mathrm{m}^{2}$ de almácigo (tabla 6). Los tratamientos solarizados tanto con PE $35 \mu \mathrm{m}$ como $80 \mu \mathrm{m}$ no tuvieron diferencias estadísticamente significativas entre sí en la cantidad de malezas presentes por metro cuadrado de cantero. Ambos fueron estadísticamente diferentes al testigo.

Las malezas predominantes en las parcelas del experimento fueron: capiquí (Stellaria media), mastuerzo (Coronopus didimus), perejilillo (Fumaria spp.), pega lana (Picris echoides), bowlesia (Bowlesia incana), falsa ortiga (Stachis arvensis), manzanilla (Matricaria chamomilla), sanguinaria (Poligonum aviculare), rábano (Raphanus sp.), cerraja (Sonchus oleraceus), "capiquí peludo" (Cerastium vulgatum) y trébol de campo (Trebol spp.). En los tratamientos solarizados la predominancia fue de trébol de campo.

Los tratamientos solarizados con PE 35 y $80 \mu \mathrm{m}$ no tuvieron diferencias estadísticamente significativas entre sí en el peso fresco, ni en el peso seco de malezas (tabla 6). Ambos fueron estadísticamente diferentes al testigo.

El análisis del contenido de nitratos y amonio en el suelo de las parcelas indicó que los tratamientos solarizados tuvieron significativamente mayor contenido de nitratos y de amonio que los no solarizados (tabla 7).

La altura de las plántulas de los tratamientos solarizados fue mayor que en el testigo (tabla 7). No se encontraron diferencias estadísticamente significativas en el diámetro del falso tallo entre los tratamientos. El peso fresco y seco de las plántulas de las parcelas solarizadas con PE 35 y 80 $\mu \mathrm{m}$ fueron las que presentaron los mayores valores, pero no fueron estadísticamente diferentes del tratamiento testigo (tabla 8). 


\begin{tabular}{|c|c|c|c|}
\hline Tratamientos & No. malezas $/ \mathrm{m}^{2}$ & Peso fresco $(\mathrm{g})$ & Peso seco (g) \\
\hline 1 No solarizado & 573 a & $1.432 \mathrm{a}$ & $256 a b$ \\
\hline 2 Solarizado con PE UV trans. $35 \mu \mathrm{m}$ & $23 \mathrm{~b}$ & $16 \mathrm{~b}$ & $4 \mathrm{c}$ \\
\hline 3 Solarizado con PE UV trans. $80 \mu \mathrm{m}$ & $54 \mathrm{~b}$ & $16 \mathrm{~b}$ & $5 c$ \\
\hline CV (\%) & 39 & 37 & 51 \\
\hline LSD & 192 & 482 & 101 \\
\hline
\end{tabular}

Los promedios con letras distintas indican diferencia significativa según la prueba de $\operatorname{LSD}(P \leq 0,01)$.

\begin{tabular}{|l|c|c|c|c|}
\hline Tabla 7. Nitratos y amonio en suelo a los $\mathbf{6 3}$ días después de levantar el polietileno de la solarización y altura \\
y diámetro del falso tallo de la plántula de cebolla a los $\mathbf{1 2 0}$ dds.
\end{tabular}

Los promedios con letras distintas indican diferencia significativa según la prueba de LSD $(P \leq 0,01)$; NS, no significativo.

\begin{tabular}{|c|c|c|c|}
\hline Tratamientos & $\begin{array}{c}\text { Peso fresco } \\
\text { (g/10 plántulas) }\end{array}$ & $\begin{array}{c}\text { Peso seco } \\
\text { (g/10 plántulas) }\end{array}$ & $\begin{array}{c}\text { Nitrógeno foliar } \\
(\%)\end{array}$ \\
\hline 1 No solarizado & $17,9 a b c$ & 2,0 & $2,96 \mathrm{bc}$ \\
\hline 2 Solarizado con PE UV trans. $35 \mu \mathrm{m}$ & $24,0 a b$ & 2,4 & $3,88 a b$ \\
\hline 3 Solarizado con PE UV trans. $80 \mu \mathrm{m}$ & $26,0 \mathrm{a}$ & 2,3 & $4,40 \mathrm{a}$ \\
\hline CV (\%) & 13 & 15 & 19 \\
\hline LSD & 6,6 & NS & 1,39 \\
\hline
\end{tabular}

Los promedios con letras distintas indican diferencia significativa según la prueba de LSD $(P \leq 0,01)$; NS, no significativo.

Las plántulas provenientes de los tratamientos con solarización presentaron un contenido de $\mathrm{N}$ foliar mayor que el tratamiento testigo (tabla 8).

En las figuras 6 y 7 se grafican los datos de temperaturas registradas a $10 \mathrm{~cm}$ de profundidad entre el 7 de diciembre de 2006 y el 28 de febrero de 2007 para el testigo sin solarizar y para los solarizados con PE 35 y $80 \mu \mathrm{m}$. Existió una diferencia muy grande en la temperatura entre los canteros solarizados y el no solarizado, con tem- peraturas cercanas y superiores a $50^{\circ} \mathrm{C}$, similares a las mencionadas como necesarias para afectar la germinación de las malezas.

El 28 de agosto (120 dds) se realizó una evaluación del estado general de los plantines utilizando una escala visual entre 1 (malo) y 5 (excelente). También se determinó en forma visual el porcentaje de punta seca de la hoja más desarrollada y el porcentaje de superficie de la misma hoja con manchas de Botrytis (tabla 9). 

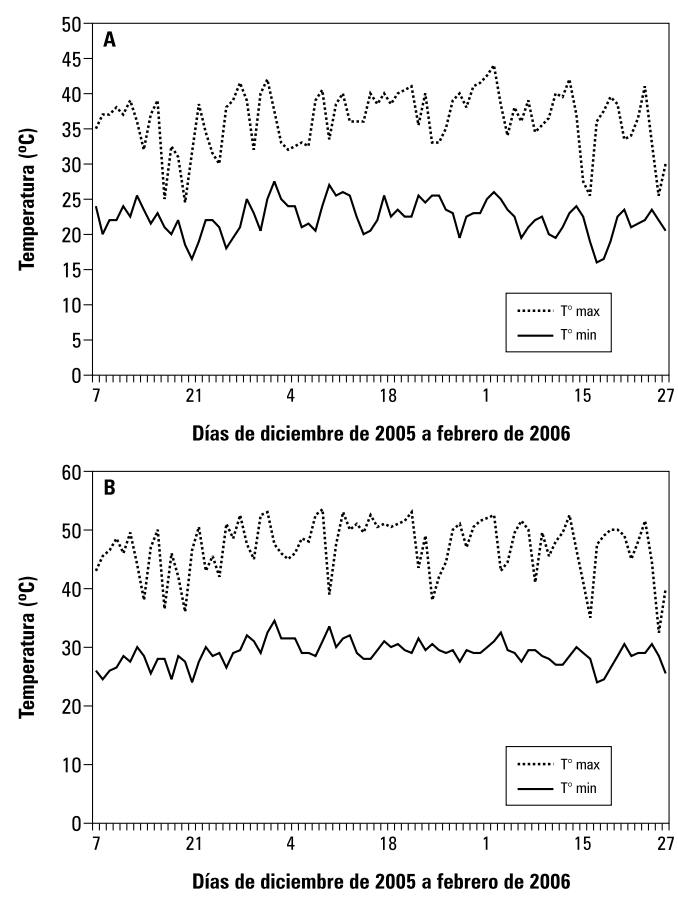

Figura 6. Temperaturas máximas y mínimas a $10 \mathrm{~cm}$ de profundidad, suelo no solarizado (A) y solarizado (B) con PE UV transp. $35 \mu \mathrm{m}$. INIA, Las Brujas, 2006-2007.

Si bien no existieron diferencias estadísticamente significativas entre las plántulas de los tratamientos solarizados y el no solarizado, las provenientes de los canteros que habían sido solarizados presentaron una tendencia a un estado general superior $(4,3$ y 5,0$)$ al de las plántulas no solarizadas (tabla 9). El porcentaje de punta seca de las plántulas solarizadas fue inferior (13 y 15\%) con relación al de las plántulas no solarizadas (23\%). Las plántulas de los canteros solarizados tendieron a un menor porcentaje de área con manchas por Botrytis (16 y 20\%) con relación a las plántulas de los canteros no solarizados (25\%).

\section{Efecto de la solarización en el control del nematodo del tallo Ditylenchus dipsaci (Kühn) Filip.}

En el periodo 2007-2008 se realizó un experimento con solarización y utilización de microorganismos efectivos (EM) en el control del nemato-

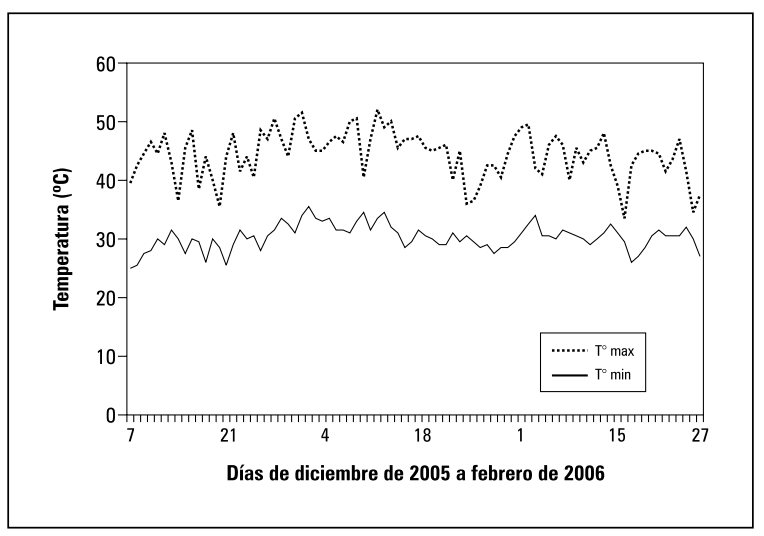

Figura 7. Temperaturas máximas y mínimas a $10 \mathrm{~cm}$ de profundidad en cantero solarizado con PE UV transp. $80 \mu \mathrm{m}$. INIA, Las Brujas, 2006-2007.

do del bulbo Ditylenchus dipsaci (Kühn) Filip. El 11 de diciembre de 2007 se colocaron los PE UV transp. $35 \mu \mathrm{m}$ en las parcelas solarizadas previo riego de las mismas hasta capacidad de campo. Se plantaron cuatro filas del cultivar Pantanoso del Sauce-CRS en canteros a $1,5 \mathrm{~m}$ y parcelas de $5 \mathrm{~m}$ de largo, el 28 de abril de 2008. Se utilizó un diseño experimental de bloques al azar con cuatro repeticiones (Arboleya et al., 2009).

Se realizó una evaluación del estado general de las parcelas 37 dds. Se usó una escala de 1 a 5 , donde $1=$ malo y $5=$ excelente. A los 39 dds se contabilizó el número de espacios sin plantas y se calculó el porcentaje de área sin plantas en 2 $\mathrm{m}$ lineales en las dos filas centrales del cantero del almácigo. Se realizó una determinación de las plantas afectadas con síntomas de nematodos a los 84 dds. Para ello se extrajeron plantas de $0,5 \mathrm{~m}$ lineales de una fila central del cantero y de $0,5 \mathrm{~m}$ lineales de una fila de afuera del cantero. Se contabilizó el número de plantas para esa superficie y se realizó una observación visual de la sintomatología de las plantas y se contó cuántas tenían síntomas visibles y cuántas no.

Posteriormente se colectaron $2 \mathrm{~g}$ de parte basal de planta se colocaron toda la noche en $50 \mathrm{~mL}$ de agua. Al otro día se colectó una muestra de $30 \mathrm{~mL}$ del fondo del agua y se centrifugó a 3.000 
Tabla 9. Evaluación sanitaria de las plántulas de cebolla a los 120 dds.

\begin{tabular}{|l|c|c|c|}
\hline \multicolumn{1}{|c|}{ Tratamientos } & Estado general & $\begin{array}{c}\text { Punta seca } \\
(\%)^{2}\end{array}$ & $\begin{array}{c}\text { Superficie foliar con } \\
\text { manchas de Botrytis (\%) }\end{array}$ \\
\hline 1 No solarizado & $3,2 \mathrm{abc}$ & $23 \mathrm{ab}$ & 25 \\
\hline 2 Solarizado con PE UV transp. $35 \mu \mathrm{m}$ & $4,3 \mathrm{ab}$ & $15 \mathrm{~b}$ & 16 \\
\hline 3 Solarizado con PE UV transp. $80 \mu \mathrm{m}$ & $5,0 \mathrm{a}$ & $13 \mathrm{~b}$ & 20 \\
\hline CV (\%) & 10 & 14 & 19 \\
\hline LSD & 0,92 & 5 & $\mathrm{NS}$ \\
\hline
\end{tabular}

${ }^{1}$ Estado 1, malo; 5, excelente; ${ }^{2}$ corregido por arcoseno raíz del porcentaje.

Los promedios con letras distintas indican diferencia significativa según la prueba de LSD $(P \leq 0,01)$; NS, no significativo.

$\mathrm{g}_{\mathrm{n}}(5.000 \mathrm{rpm})$. Se tomaron los $5 \mathrm{~mL}$ del fondo y se contó el número de nematodos en esos $5 \mathrm{~mL}$. Esto se realizó en plantas con y sin síntomas visuales de nematodos.

El estado general de las parcelas del testigo fue significativamente inferior al de los tratamientos solarizados que no difirieron entre sí (tabla 10). La misma tendencia se observó en los espacios sin plantas entre el testigo y los solarizados. El número de plantas afectadas con síntomas visuales de nemátodos fue significativamente superior en el tratamiento sin solarizar (tabla 11).

La cantidad de nematodos en plantas con síntomas visuales de esta enfermedad detectada en el tratamiento testigo fue muy superior al de la registrada en las plantas con síntomas en los otros tratamientos, a pesar de no haber diferencias significativas, debido seguramente al elevado coeficiente de variación registrado (tabla 12).

Debe destacarse que en las plantas que visualmente no tenían síntomas y estaban dentro del área en donde se extrajeron plantas con síntomas $(0,5 \mathrm{~m}$ lineales) también se detectaron nematodos siendo sensiblemente mayor y estadísticamente significativas esas diferencias en el caso del tratamiento testigo.

\section{CONCLUSIONES GENERALES}

La solarización ha sido efectiva en reducir el banco de semillas de malezas bajo diferentes condiciones y localidades.

Tabla 10. Observación del estado general de las parcelas a los 37 dds y número de espacios y área sin plántulas de cebolla a los 39 dds.

\begin{tabular}{|l|c|c|c|}
\hline \multicolumn{1}{|c|}{ Tratamientos } & $\begin{array}{c}\text { Estado general } \\
\text { de las parcelas }\end{array}$ & $\begin{array}{c}\text { No. de espacios } \\
\text { sin plantas }\end{array}$ & $\begin{array}{c}\text { Área sin plantas } \\
(\%)\end{array}$ \\
\hline 1 Testigo sin solarizar & $1,5 \mathrm{~b}$ & $10,0 \mathrm{a}$ & $33,0 \mathrm{a}$ \\
\hline 2 Solarizado con PE UV transp. 35 $\mu \mathrm{m}$ & $4,5 \mathrm{a}$ & $0,7 \mathrm{~b}$ & $1,1 \mathrm{~b}$ \\
\hline $\begin{array}{l}3 \text { Solarizado con PE UV transp. 35 } \mu \text { m agregando } 200 \mathrm{~L} \mathrm{ha}^{-1} \text { de EM al } \\
\text { momento de la solarización }\end{array}$ & $4,0 \mathrm{a}$ & $1,5 \mathrm{~b}$ & $1,5 \mathrm{~b}$ \\
\hline $\begin{array}{l}\text { 4 Solarizado con PE UV transp. 35 } \mu \text { m agregando 200 } \mathrm{L} \mathrm{ha}^{-1} \text { de EM al } \\
\text { momento de la siembra y cada 20 días }\end{array}$ & $4,5 \mathrm{a}$ & $1,0 \mathrm{~b}$ & $1,1 \mathrm{~b}$ \\
\hline CV (\%) & 22 & 65 & 148 \\
\hline LSD & 1,84 & 5,53 & 21,0 \\
\hline
\end{tabular}

${ }^{1}$ Estado 1, malo; 5, excelente; ${ }^{2}$ corregido por arcoseno raíz del porcentaje.

Los promedios con letras distintas indican diferencia significativa según la prueba de $\operatorname{LSD}(P \leq 0,01)$.

REV. COLOMB. CIENC. HORTIC. 
Tabla 11. Plantas de cebolla afectadas por síntomas visuales de nematodos a los 84 dds.

\begin{tabular}{|c|c|c|c|c|}
\hline Tratamientos & $\begin{array}{l}\text { No. de plantas } \\
\text { en } 0,5 \mathrm{~m} \text { en la } \\
\text { fila de afuera } \\
\text { del cantero }\end{array}$ & $\begin{array}{l}\text { Plantas con } \\
\text { síntomas } \\
\text { visuales en la } \\
\text { fila de afuera } \\
\text { del cantero (\%) }\end{array}$ & $\begin{array}{l}\text { No. de plantas } \\
\text { en } 0,5 \mathrm{~m} \text { en la } \\
\text { fila de adentro } \\
\text { del cantero }\end{array}$ & $\begin{array}{l}\text { Plantas con } \\
\text { síntomas } \\
\text { visuales en la } \\
\text { fila de adentro } \\
\text { del cantero (\%) }\end{array}$ \\
\hline 1 Testigo sin solarizar & $25 \mathrm{~b}$ & $74 \mathrm{a}$ & $40 \mathrm{~b}$ & $74 \mathrm{a}$ \\
\hline 2 Solarizado con PE UV transp. $35 \mu \mathrm{m}$ & $94 \mathrm{a}$ & $19 \mathrm{~b}$ & 103 a & $4 \mathrm{~b}$ \\
\hline $\begin{array}{l}3 \text { Solarizado con PE UV transp. } 35 \mu \text { m agregando } 200 \mathrm{~L} \\
\text { ha-1 de EM al momento de la solarización }\end{array}$ & 95 a & $12 \mathrm{~b}$ & 92 a & $5 b$ \\
\hline $\begin{array}{l}4 \text { Solarizado con PE UV transp. } 35 \mu \mathrm{m} \text { agregando } 200 \mathrm{~L} \\
\text { ha-1 de EM al momento de la siembra y cada } 20 \text { días }\end{array}$ & 107 a & $15 b$ & $110 \mathrm{a}$ & $4 \mathrm{~b}$ \\
\hline CV (\%) & 28 & 39 & 23 & 20 \\
\hline LSD & 52 & 31 & 45 & 11 \\
\hline
\end{tabular}

Los promedios con letras distintas indican diferencia significativa según la prueba de LSD $(P \leq 0,01)$.

Tabla 12. Número de nematodos en plantas de cebolla con y sin síntomas visibles a los 84 dds.

\begin{tabular}{|l|c|c|}
\multicolumn{1}{|c|}{ Tratamientos } & $\begin{array}{c}\text { No. de nematodos en plantas con } \\
\text { síntomas visuales de nematodos }\end{array}$ & $\begin{array}{c}\text { No. de nematodos en plantas sin } \\
\text { síntomas visuales de nematodos }\end{array}$ \\
\hline 1 Testigo sin solarizar & 159 & $31 \mathrm{a}$ \\
\hline 2 Solarizado con PE UV transp. 35 $\mu \mathrm{m}$ & 49 & $2 \mathrm{~b}$ \\
\hline $\begin{array}{l}3 \text { Solarizado con PE UV transp. 35 } \mu \text { m agregando } \\
200 \text { L ha }^{-1} \text { de EM al momento de la solarización }\end{array}$ & 34 & $1 \mathrm{~b}$ \\
\hline $\begin{array}{l}4 \text { Solarizado con PE UV transp. 35 } \mu \text { m agregando } \\
200 \text { ha }^{-1} \text { de EM al momento de la siembra y cada } \\
20 \text { días }\end{array}$ & 35 & $0 \mathrm{~b}$ \\
\hline CV (\%) & 106 & 129 \\
\hline LSD & NS & 25,5 \\
\hline
\end{tabular}

Los promedios con letras distintas indican diferencia significativa según la prueba de LSD $(P \leq 0,01)$; NS, no significativo.

El uso de PE UV transp. $35 \mu \mathrm{m}$ fue tan efectivo en reducir el banco de semillas de malezas como el de $80 \mu \mathrm{m}$. La calidad de la plántula fue similar con el uso de ambos espesores de polietileno.

El uso de la solarización demostró tener un beneficio adicional y favorable sobre el comportamiento sanitario en el almácigo, al reducir el ambiente de humedad que se genera con la presencia de malezas y que favorecen el desarrollo de los patógenos.
La solarización incrementa los niveles de nitratos $y$ de amonio en el suelo por lo que debe tenerse en cuenta para no realizar aplicaciones innecesarias de $\mathrm{N}$, que pueden traer efectos negativos sobre el desarrollo de los plantines y favorecer el ataque de enfermedades.

Fue claro el efecto favorable de la solarización en la reducción de la sintomatología ocasionada por los nematodos y en obtener plántulas de mejor calidad. 


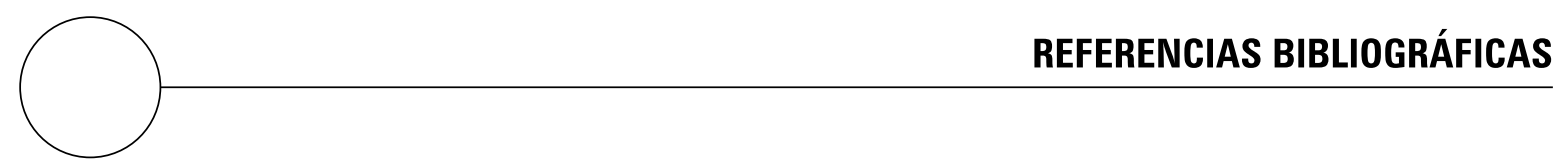

Arboleya, J.; D. Maeso; E. Campelo; M. Falero y W. Walasek. 2009. Manejo integrado de almácigos de cebolla. Investigación aplicada. INIA-DIGEGRAPPR -MGAP. Efecto de la solarización de canteros en el control de nemátodos en almácigos de cebo1la. pp. 75-85. En: Jornada Anual de Presentación de Resultados de Investigación en Cebolla. Serie Actividades de Difusión No. 564. INIA Las Brujas, Rincón del Colorado, Uruguay.

Arboleya, J.; J. Rodríguez y E. Campelo. 2008. Alternativas al control químico de malezas en almácigos de cebolla. Solarización de canteros para almácigos. pp. 29-40. En: Jornada Anual de Presentación de Resultados de Investigación en Cebolla y Ajo. Serie Actividades de Difusión No. 533. INIA Las Brujas, Rincón del Colorado, Uruguay.

Campelo, E. y J. Arboleya. 2005. Actualidad de la producción de cebolla en Uruguay. pp 1-15. En: Arboleya, J. (ed.) Tecnología para la producción de cebolla. Boletín de Divulgación 88. INIA Las Brujas, Rincón del Colorado, Uruguay.

Campelo, E.; J. Arboleya y J. Rodríguez. 2006. Solarización de canteros para almácigos de cebolla. Serie Actividades de Difusión No. 466. INIA Las Brujas, Rincón del Colorado, Uruguay.

Campelo, E.; J. Arboleya y J. Rodríguez. 2007. Módulos de manejo integrado en almácigos de cebolla. Solarización de canteros para almácigos. En: Resultados Experimentales en Ajo y Cebolla. Serie Actividades de Difusión No. 482. INIA Las Brujas Rincón del Colorado, Uruguay.

Davis, J.R. 1991. Soil solarization: pathogen and disease control and increases in crop yield and quality: short- and long term effects and integrated control. pp. 39-50. En: Katan J. y J.E. De Vay (eds.). Soil solarization. CRC Press, Boca Raton, FL.

DIEA-MGAP, Dirección de Estadísticas Agropecuarias Ministerio de Ganadería, Agricultura y Pesca. 2008. Encuestas hortícolas 2007. Zona sur y litoral norte. Serie Encuestas No. 263. Uruguay.

El Clima del Uruguay. Características climáticas del Uruguay. Variables climáticas. Dirección General de Meteorología. En: http://www.meteorologia.com. uy/caract_climat.htm\#; consulta: julio de 2009.

Gil, R.; A. Smith; B. Chaves; K. Wyckhuys; C. Forero y J. Jiménez. 2009. Combined efficazy assessment of soil solarization and bio-fungicides for management of Sclerotinia spp. in lettuce (Lactuca sativa L.). Agron. Colomb. 27(2), 193-201.

Hartmann, H.T.; D.E. Kester; F.E. Davies y R. Geneve. 2001. Hartmann and Kester's plant propagation: Principles and practices. $7^{\text {th }}$ ed. Prentice Hall, Upper Saddle River, New Jersey, NJ.

Katan, J.; A. Greenberger; H. Alon y A. Grinstein. 1976. Solar heating by polyethylene mulching for the control of diseases caused by soilborne pathogens. Phytopathol. 66, 683-688.

Mallek, S.B.; T.S. Prather y J.J. Stapleton. 2007. Interaction effects of Allium sp., concentrations and soil temperature on seed germination of four weedy pant species. Appl. Soil Ecol. 37, 233-239.

Severota, V. 1997. Características generales del clima sobre Uruguay. En: http://www.rau.edu.uy/uruguay/ geografia/Uy_c-info.htm; consulta: julio de 2009. 